\title{
Virtual Reality and Its Effectiveness on Motor Development and Rehabilitation in Children with Disorders
}

\author{
Brent Brooke ${ }^{1}$, Tao Zhang ${ }^{1 *}$, Joonyoung Lee ${ }^{1}$, Xiangli Gu${ }^{2}$ and Ana West ${ }^{1}$ \\ ${ }^{1}$ Department of Kinesiology, Health Promotion and Recreation, University of North Texas, Denton, USA \\ ${ }^{2}$ Department of Kinesiology, University of Texas at Arlington, USA \\ *Corresponding author: Tao Zhang, Department of Kinesiology, Health Promotion and Recreation, University of North Texas, Denton, \\ USA. \\ To Cite This Article: Tao Zhang, Virtual Reality and Its Effectiveness on Motor Development and Rehabilitation in Children with Disorders. 2020 \\ - 7(3). AJBSR.MS.ID.001156. DOI: 10.34297/AJBSR.2020.07.001156.
}

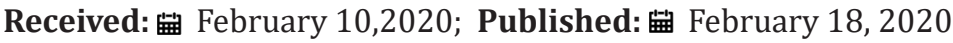

\begin{abstract}
Recent advancements in Virtual Reality (VR) technology has opened a wide array of its practical uses in rehabilitation, physical activity, and motor skill acquisition among children. During VR simulations, participants practice specific movements and movement patterns, in a computersimulated environment that emulates the real world. However, to date, limited suggestions about the effectiveness and feasibility of VR technology in children with disorders have been informed. This mini review aimed to identify the most effective VR systems being used in pediatric rehabilitation and examine the effects of VR on physical activity and motor competence of children with disorders over time. Recent VR studies and literature reviews were examined in order to identify these effects.
\end{abstract}

Results showed that VR is useful and efficacious in rehabilitation, especially to children with disorders such as developmental coordination disorder, cerebral palsy, hemiplegia, Down syndrome, and developmental dyspraxia. The findings indicated that VR could potentially increase the enjoyment of physical activities that contribute to time spent being physically active daily. However, there is a limited amount of research evidence concerning its effectiveness and feasibility of VR on motor competence among children. Future research should expand on the long-term effects of physical activity and motor development among children with disorders as well as the efficacy of different VR systems when pediatric rehabilitation is concerned.

Keywords: Virtual reality, Rehabilitation, Motor learning, Motor skill acquisition, Physical activity, Children, Disorders

\section{Introduction}

Virtual Reality (VR) is a unique and useful tool that researchers and practitioners use when motor learning, movement deficiencies, and rehabilitation are concerned [1]. During VR, the user interacts with a multidimensional computer-generated environment that can be explored in real-time, which is a vital feature of the functional use of VR [2]. For the past decades, technology has become increasingly prevalent in the lives of children. Previous studies suggested VR video games using Nintendo Wii and Sony Eye-Toy promote physical activity in school-aged children [3]. Lu et al. [4] suggested that active video games increase movement and physical activity levels by allowing players to physically interact with images on-screen in a range of activities by using arm, leg or whole-body movements and scan aid in the prevention of childhood obesity. Earlier VR research had focused specifically on comparing movements made with, or to, virtual objects in a virtual environment and compares them to movements made with or to real objects in real environments [5].

Immersive and non-immersive VR systems such as the Nintendo Wii, Sony Eye-Toy, and XBOX Kinect, were some of the most common systems used in recent research [6]. In recent years, the popularity of VR being used in clinical and research settings has expanded exponentially as new technological advancements have been made, especially when the fields of physical activity and pediatric rehabilitation are concerned [7]. Recently, researchers and practitioners have begun using immersive VR headsets such 
as the HTC-Vive, Oculus Rift, and PlayStation VR more than the console-based non-immersive game systems like the Sony Eye-Toy, Xbox Kinect, and Nintendo Wii. The HTC-Vive seems to be one of the most popular systems currently used by practitioners as it brought needed innovations in terms of the controller, the front camera, and the display with its high resolution, large field of view, and high refresh rate [8].

Systems like the Oculus Rift are not as commonly used in rehabilitation and research when compared to the HTC-Vive, however they are still compatible with the older consoles like the Xbox Kinect and have a strong presence when the promotion of physical activity is concerned, especially in pediatric populations [9]. Regardless of which console or system is used, researchers have found that games and programs available on these systems are "entertaining" and distracting to the participants [10]. This is due in part to the participant focusing on the gameplay rather than his or her impairment, which in turn results in more enjoyable physical activity and improved adherence to and completion of fitness exercise and rehabilitation training [10]. However, given the fact that the VR field itself is relatively new, there is a dearth of research concerning its effects and functionality among children with motor skill deficiencies or other disabilities such as developmental coordination disorder, cerebral palsy, hemiplegia, Down syndrome, and developmental dyspraxia. Thus, more research is needed to explore potential benefits that VR offers [11].

\section{The Effects of VR on Motor Competence in Healthy Children}

It is evident that regular participation in physical activity is associated with important short- and long-term physiological and psychological health benefits in children [12]. Unfortunately, the amount of physical activity children participate in is decreasing over time and can contribute to the early onset of health problems such as obesity, heart disease, and Type 2 diabetes [13]. From a public health perspective, children with delay or deficit in motor development are often at elevated risk of physical inactivity and hence may be referred to as a vulnerable population [14]. Lack of physical activity at a young age may contribute to poor motor competence in life or a delay in their motor development, so physical activity interventions are urgently needed among schoolaged children [13]. Additionally, a systematic review concluded that physical activity interventions improved children's movement skills, which commonly had large effect sizes in typically developing children and could contribute to an increase in physical activity [15]. Ericsson and Karlsson [16] supported this notion that physical activity interventions, through the use of VR, improve movement skill and could contribute to an increased physical activity among children.

Perceived motor competence plays an important role in fostering children's physical activity [17]. In addition, children who may not have been physically active earlier on in their lives may be lacking of their fundamental motor skills [13]. The VR games in general, have been shown to be an effective preventative approach in increasing physical activity levels among inactive children [18]. Research suggested that VR may promote early development of motor competence in children akin to more natural, real-world development as opposed to strictly formalized training. Kiefer and colleagues [13] also suggest that the initiation of VR training at an earlier age in children relates to an increased likelihood of avoiding physical inactivity later in life.

Learning fundamental movement skills in a virtual environment would be an especially effective and feasible approach to improve motor competence and overall physical activity levels among children. Previous studies showed that an increase in children's motor competence is positively correlated to improved physical activity and sport participation [19]. However, there is a limited amount of research that focuses on whether active video games have additional benefits such as increasing motor development, particularly in young children [13]. This lack of research may be due to negative side effects that may present themselves while the participant is engaged is VR such as dizziness, injury, and disorientation. Because VR studies involving children tend to have smaller sample sizes and are not commonly longitudinal, the effect of VR on physical activity and motor competence need to be examined in much greater detail.

\section{The Effects of VR on Motor Competence in Children with Disorders}

\section{Developmental Coordination Disorder}

Developmental Coordination Disorder (DCD) refer to fine and gross motor problems of varying levels of severity [20], which affects between $5 \%$ and $10 \%$ of children worldwide. Children with DCD do not have an identifiable medical or neurological condition that explains their coordination problems; however, VR may serve as a diagnostic tool to determine the severity of DCD [21]. For example, Yeger E et al. [21] collective research identified VR as a diagnostic tool. The findings of their study suggested that VR may not be able to significantly reduce the severe motor skill limitations of children with DCD, however, it is useful as a diagnostic tool that measures actual motor skills.

As a good case in point, VR-based physical activity video games have recently been found to be useful training tools for children with DCD as they are functional and allow the implementation of various and specific movement types [22]. Bonney and colleagues [22] implemented these various practice methods and suggested that playing VR-based physical activity games provides positive transfer effects on balance tasks, running and agility tasks, and functional activities such as long jump and stair climbing. They suggested that the transfer of motor skills from VR to the real-world were not better for participants in a variable practice schedule 
compared to participants in the repetitive protocols. However, other studies examining the transfer of motor skills from VR to the real world suggest that its use improves the accuracy of both motor performance and learning [22].

\section{Cerebral Palsy}

Cerebral Palsy (CP) is a non-progressive neurological disorder appearing in infancy or early childhood that permanently effects the sensory motor capabilities of those afflicted with it [23]. While there are already a lot of rehabilitation methods that contribute to increasing the motor competence of children with $\mathrm{CP}$, the most effective methods should involve task engagement and participant enjoyment, which can be achieved through VR [24]. Other researchers have suggested that in general VR interventions showed a strong effect in improving motor function in children with CP [25]. However, it is still uncertain if a VR-based cognitive behavioral therapy would be more effective than conventional methods [26]. Thus, research involving CP and VR should focus primarily on improving the movements and motor skills involving the upper extremities in young children as well as comparing the efficacy of VR as a form of cognitive behavioral therapy to more conventional methods.

\section{Hemiplegia}

Hemiplegia is a neurological symptom appearing on the unilateral arm and leg of the body that causes affected upper/lower limb muscle weakening and difficulties in body coordination [27]. Among those with cerebral palsy, hemiplegia may present itself as a severe symptom affecting individuals' movement. VR has been a recently introduced method used to improve body coordination, strengthen limbs, and improve overall motor skill competence in children with hemiplegia [28]. Recent research showed that implementation of VR tasks involving bilateral hand and upper limb exercises can positively and significantly affect the overall performance of upper limb motor skill tasks and children' bilateral hand coordination [29].

\section{Down Syndrome}

Down Syndrome (DS) is a genetic disorder characterized by having one additional chromosome. Researchers reported that individuals with DS have less functional performance and have abnormal alterations in cognitive, motor areas, and social function [30]. Because of these abnormalities, research has focused on identifying the severity of each of these dysfunctions using a litany of techniques and measures. VR has become increasingly popular due to its ability to change the environment participants perceives themselves to be in and because of its functionality in rehabilitation with other mental and physical disorders [7]. VR is especially useful when motor learning and training is concerned due to the fact it is easily applicable and allows the participant to engage in a variable number of practice methods. For example, Monteiro M et al. [31] took these practice methods into account when they studied how those affected by DS learn and perform a variation of tasks when these different methods are used.

The findings suggested that participants in a blocked practice schedule performed significantly worse during a retention-based coincidence timing task when compared to those who were tested on a random or variable practice schedule. Another study included two different groups of children with DS, one group used VR for their rehabilitation, and the other group used a traditional occupational therapy respectively. Results of this study indicated that VR improved motor proficiency, visual-integrative abilities, and sensory integrative functions for children with DS just as much as those who participated in the traditional approach [32].

\section{Developmental Dyspraxia}

Developmental Dyspraxia (DD) is a neurodevelopmental disorder characterized by an impairment in the ability to plan and carry out sensorimotor tasks, much like cerebral palsy, and effects $6 \%$ of children [33]. Children with DD are greatly affected by their inability to participate in physical activity and have lower levels of perceived motor competence in which could be improved or benefitted by the use of VR [34]. Rehabilitation methods are recommended to take place in an attractive setting, one that can be provided through VR, as well as being intense in order to promote the reorganization of neural networks, thus improving motor development and learning [35]. Practitioners' ability to customize the virtual environment make VR an effective and important rehabilitation tool, because of its ability to be specifically tailored to the participant and because it may motivate the child to be more engaged as it can make an intense or strenuous task enjoyable [36].

\section{Practical Implications and Future Direction}

The use of VR in motor learning and rehabilitation is increasing as new and better technology is released, whether they are objective measures created by practitioners in their labs, or through new games or consoles released by companies like Nintendo etc. VR is seemingly growing in popularity as a rehabilitation method for those who suffer from physical or mental disorders. The translation of these skills, practiced in a virtual environment, is more noticeable than tasks involving motor learning or perceived/ actual motor competence because they are much more specific than those concerning skill acquisition, especially in normally developed, healthy children. It is evident that VR can be used as a useful diagnostic tool that objectively measures performance of certain skills or tasks especially in children.

VR is particularly useful not only as a diagnostic tool, but also as a tool for rehabilitation and education among those who suffer from diseases or disorders, such as developmental coordination disorder, cerebral palsy, hemiplegia, Down syndrome, and developmental dyspraxia. Practitioners and researchers are turning to VR as a 
rehabilitation tool as well to increase physical activity and motor development among children who are inactive or are physically compromised.

\section{Potential Limitations}

Although VR is a promising technology in the physical activity and rehabilitation field, more research is needed when the age of children who use VR is concerned. There is lack of research regarding the appropriate age VR should be introduced and the length of time children should be exposed to it. Though the use of VR is becoming more prevalent, there is still a dearth of research examining how the skills practiced or learned in these virtual environments translate to the real world over time. More research is needed to examine the relationship between the use of VR and its effect on improving physical activity and motor development in children with disorders.

\section{Conclusion}

It is evident that VR is useful and efficacious in rehabilitation, especially to children with disorders such as developmental coordination disorder, cerebral palsy, hemiplegia, Down syndrome, and developmental dyspraxia. VR could potentially increase the enjoyment of physical activities that contribute to time spent being physically active daily. Future research should examine the efficacy of VR on motor development and physical activity in children with disorders when pediatric rehabilitation is concerned.

\section{References}

1. Osumi M, Inomata K, Inoue Y, Otake Y, Morioka, et al. (2019) Characteristics of Phantom Limb Pain Alleviated with Virtual Reality Rehabilitation. Pain Med 20(5): 1038-1046.

2. Minderer M, Harvey CD, Donato F, Moser EI (2016) Neuroscience: Virtual Reality Explored. Nature 533(7603): 324-325.

3. Marcos DP (2018) Virtual Reality Experiences, Embodiment, Videogames and Their Dimensions in Neurorehabilitation. J NeuroEng Rehabil 15(1): 113.

4. Lu AS, Baranowski T, Hong SL, Buday R, Thompson D, et al. (2016) The Narrative Impact of Active Video Games on Physical Activity among Children: A Feasibility Study. J Med Internet Res 18(10): 272.

5. Viau A, Feldman AG, Mc Fadyen BJ, Levin MF (2004) Reaching in reality and virtual Reality: A Comparison of Movement Kinematics in Healthy Subjects and in Adults with Hemiparesis. J Neuro Eng Rehabil 1(1): 11.

6. Duncan M, Staples V (2010) The Impact of a School-Based Active Video Game Play Intervention on Children's Physical Activity during Recess. Hum Mov 11(1): 95-99.

7. Howard MC (2017) A Meta-Analysis and Systematic Literature Review of Virtual Reality Rehabilitation Programs. Comput Human Behav 70: 317-327.

8. Niehorster DC, Li Li, Lappe M (2017) The Accuracy and Precision of Position and Orientation Tracking in the HTC Vive Virtual Reality System for Scientific Research. Iperception 8(3).

9. Trombetta M, Henrique PPB, Brum MR, Colussi EL, De Marchi ACB, et al. (2017) Motion Rehab AVE 3D: A VR-Based Exergame for Post-Stroke Rehabilitation. Comput Methods Programs Biomed 151: 15-20.
10. Taylor MJ, Mc Cormick D, Shawis T, Impson R, Griffin M (2011) ActivityPromoting Gaming Systems in Exercise and Rehabilitation. J Rehabil Res Dev 48(10): 1171-1186.

11. Boone A, Engsberg J (2016) Introduction of a Virtual Reality Motor Rehabilitation Intervention and Perceptions of Feasibility from Key Stakeholders. Arch Phys Med Rehabil 97(10): 89-90.

12. Carlsson E, Ludvigsson J, Huus K, Faresjo M (2015) High Physical Activity in Young Children Suggests Positive Effects by Altering AutoantigenInduced Immune Activity. Scand J Med Sci Sport 26(4): 441-450.

13. Kiefer AW, Pincus D, Richardson MJ, Myer GD (2017) Virtual Reality as a Training Tool to Treat Physical Inactivity in Children. Front Public Heal 5: 349.

14. Barr G, Zelazo PR, Zelazo PD (2014) Challenges to Developmental Paradigms: Implications for Theory, Assessment and Treatment. $1^{\text {st }} \mathrm{Ed}$, Taylor \& Francis Hoboken NJ, New York, USA pp.312.

15. Robinson LE, Stodden DF, Barnett LM, Lopes VP, Logan SW, et al. (2015) Motor Competence and Its Effect on Positive Developmental Trajectories of Health. Sport Med 45(9): 1273-1284.

16. Ericsson I, Karlsson M (2012) Motor Skills and School Performance in Children with Daily Physical Education in School - A 9 Year Study. Scand J Med Sci Sport 24: 273-278.

17. Meester AD, Stodden D, Brian A, True L, Cardon G, et al. (2016) Associations among Elementary School Children's Actual Motor Competence, Perceived Motor Competence, Physical Activity and BMI: A Cross-Sectional Study. PLoS One 11(10): 0164600.

18. Lubans DR, Morgan PJ, Cliff DP, Barnett LM, Okely AD (2010) Fundamental Movement Skills in Children and Adolescents: Review of Associated Health Benefits. Sport Med 40(12): 1019-1035.

19. Rosa RL, Ridgers ND, Barnett LM (2013) Development and Use of an Observation Tool for Active Gaming and Movement (OTAGM) to Measure Children's Movement Skill Components during Active Video Game Play. Percept Mot Skills 117(3): 935-949.

20. Gibbs J, Appleton J, Appleton R (2007) Dyspraxia or Developmental Coordination Disorder? Unravelling the Enigma. Arch Disabl Child 92(6): 534-539.

21. Engel Yeger B, Sidon R, Mimouni Bloch A, Weiss PL (2017) Relationship between Perceived Competence and Performance during Real and Virtual Motor Tasks by Children with Developmental Coordination Disorder. Assist Technol 12(7): 752-757.

22. Bonney E, Jelsma LD, Ferguson GD, Smits Engelsman BC (2017). Learning Better by Repetition or Variation? Is Transfer at Odds with Task Specific Training? PLoS One 12(3): 1-17.

23. Rosenbaum P, Paneth N, Leviton A, Goldstein M, Bax M, et al. (2007) A Report: The Definition and Classification of Cerebral Palsy April 2006. Dev Med Child Neurol 109: 8-14.

24. Weiss PL, Tirosh E, Fehlings D (2014) Role of Virtual Reality for Cerebral Palsy Management. J Child Neurol 29(8): 1119-1124.

25. Chen Y, Fanchiang HD, Howard A (2018) Effectiveness of Virtual Reality in Children with Cerebral Palsy: A Systematic Review and Meta-Analysis of Randomized Controlled Trials. Phys Ther 98(1): 63-77.

26. Maples K J L, Bunnell BE, Kim SJ, Rothbaum BO (2017) The Use of Virtual Reality Technology in the Treatment of Anxiety and Other Psychiatric Disorders. Harv Rev Psychiatry 25(3): 103-113.

27. Cooper J, Majnemer A, Rosenblatt B, Birnbaum R (1995) The Determination of Sensory Deficits in Children with Hemiplegic Cerebral Palsy. J Child Neurol 10(4): 300-309.

28. Green D, Wilson PH (2012) Use of Virtual Reality in Rehabilitation of Movement in Children with Hemiplegia-A Multiple Case Study Evaluation. Disabil Rehabil 34(7): 593-604. 
29. Do J, Yoo E, Jung MY, Park H (2016) The Effects of Virtual-Reality Based Bi-Lateral Arm Training on Hemiplegic Children's Upper-Arm Limb Motor Skills. Neural Rehabil 38(2): 115-127.

30. Latash ML, Kang N, Patterson D (2002) Finger Coordination in Persons with Down Syndrome: Atypical Patterns of Coordination and the Effects of Practice. Exp brain Res 146(3): 345-355.

31. De Mello MCB, Massetti T, Da Silva TD, Van Der KJ, De Abreu LC, et al. (2014) Transfer of Motor Learning from Virtual to Natural Environments in Individuals with Cerebral Palsy. Res Dev Disabil 35(10): 2430-2437.

32. Wuang YP, Chiang CS, Su CY, Wang CC (2011) Effectiveness of Virtual Reality Using Wii Gaming Technology in Children with Down Syndrome. Res Dev Disabil 32(1): 312-321.
33. Bortone I, Leonardis, D, Mastronicola N, Crecchi A, Bonfiglio L, et al. (2018) Wearable Haptics and Immersive Virtual Reality Rehabilitation Training in Children with Neuromotor Impairment. IEEE Trans Neural Syst Rehabil Eng 26(7): 1469-1478.

34. Bodison SC (2015) Developmental Dyspraxia and the Play Skills of Children with Autism. Am J Occup Ther 69(5): 1-6.

35. Smits EC, Blank R, Van Der KA, Mosterd Van DMR, Vlugt Van DBE, et al. (2012) Efficacy of Interventions to Improve Motor Performance in Children with Developmental Coordination Disorder: A Combined Systematic Review and Meta-analysis. Dev Med Child Neurol 55(3): 229237.

36. Slater M, Sanchez Vives MV (2016) Enhancing Our Lives with Immersive Virtual Reality. Front Robot AI 3: 74. 